\title{
Equilibrium Kawasaki dynamics of continuous particle systems
}

\author{
Yuri Kondratiev \\ Fakultät für Mathematik, Universität Bielefeld, Postfach 1001 31, D-33501 Bielefeld, \\ Germany; BiBoS, Univ. Bielefeld, Germany. \\ e-mail: kondrat@mathematik. uni-bielefeld.de \\ Eugene Lytvynov \\ Department of Mathematics, University of Wales Swansea, Singleton Park, Swansea \\ SA2 8PP, U.K. \\ e-mail: e.lytvynov@swansea.ac.uk \\ Michael Röckner \\ Fakultät für Mathematik, Universität Bielefeld, Postfach 1001 31, D-33501 Bielefeld, \\ Germany; BiBoS, Univ. Bielefeld, Germany. \\ e-mail: roeckner@mathematik. uni-bielefeld.de
}

\begin{abstract}
We construct a new equilibrium dynamics of infinite particle systems in a Riemannian manifold $X$. This dynamics is an analog of the Kawasaki dynamics of lattice spin systems. The Kawasaki dynamics now is a process where interacting particles randomly hop over $X$. We establish conditions on the a priori explicitly given symmetrizing measure and the generator of this dynamics, under which a corresponding conservative Markov processes exists. We also outline two types of scaling limit of the equilibrium Kawasaki dynamics: one leading to an equilibrium Glauber dynamics in continuum (a birth-and-death process), and the other leading to a diffusion dynamics of interacting particles (in particular, the gradient stochastic dynamics).
\end{abstract}

2000 AMS Mathematics Subject Classification: 60K35, 60J75, 60J80, 82C21, 82C22

Keywords: Birth-and-death process; Continuous system; Gibbs measure; Glauber dynamics; Gradient stochastic dynamics; Kawasaki dynamics; Scaling limit.

\section{Introduction}

In the classical $d$-dimensional Ising model with spin space $S=\{-1,1\}$, the Kawasaki dynamics means that pairs of neighboring particles with different spins randomly exchange their spin values. The generator of this dynamics is given by

$$
(H f)(\sigma)=\sum_{x \in \mathbb{Z}^{d}} \sum_{y \in \mathbb{Z}^{d},|x-y|=1} c(x, y, \sigma)\left(\nabla_{x y} f\right)(\sigma)
$$


where

$$
\left(\nabla_{x y} f\right)(\sigma)=f\left(\sigma^{x y}\right)-f(\sigma),
$$

$\sigma^{x y}$ denoting the configuration $\sigma$ in which the particles at sites $x$ and $y$ have exchanged their spin values. Under appropriate conditions on the coefficient $c(x, y, \sigma)$, the corresponding dynamics has a Gibbs measure as symmetrizing (hence invariant) measure. We refer, e.g., to [24] for a discussion of the Kawasaki dynamics of lattice spin systems.

Let us now interpret a lattice system with spin space $S=\{-1,1\}$ as a model of a lattice gas. Then $\sigma(x)=1$ means that there is a particle at site $x$, while $\sigma(x)=-1$ means that the site $x$ is empty. The Kawasaki dynamics of such a system means that particles randomly hop from one site to another.

If we consider a continuous particle system, i.e., a system of particles which can take any position in the Euclidean space $\mathbb{R}^{d}$, then an analog of the Kawasaki dynamics should be a process in which particles randomly hop over the space $\mathbb{R}^{d}$. The generator of such a process is informally given by

$$
(H F)(\gamma)=\sum_{x \in \gamma} \int_{\mathbb{R}^{d}} c(x, y, \gamma)\left(D_{x y}^{-+} F\right)(\gamma) d y
$$

where

$$
\left(D_{x y}^{-+} F\right)(\gamma)=F(\gamma \backslash x \cup y)-F(\gamma)
$$

and the coefficient $c(x, y, \gamma)$ describes the rate at which the particle $x$ of the configuration $\gamma$ jumps to $y$. Here and below, for simplicity of notations, we just write $x$ instead of $\{x\}$.

In [12], Glötzl considered the formal generator (1.1) and derived conditions on the coefficient $c(x, y, \gamma)$ under which the operator (1.1) becomes symmetric in the space $L^{2}(\mu)$, where $\mu$ is a given Gibbs measure. However, till now, there has been no proof of the very existence of a Kawasaki dynamics of an infinite system of interacting particles in continuum.

Thus, the aim of this paper is to present a general theorem on the existence of an equilibrium Kawasaki dynamics of a continuous particle system, which has a Gibbs measure as symmetrizing (hence invariant) measure. We shall also consider some examples of such a dynamics. Finally, we shall outline two types of scaling limit of the equilibrium Kawasaki dynamics: one leading to an equilibrium Glauber dynamics in continuum (a birth-and-death process), and the other leading to a diffusion dynamics of interacting particles (in particular, the gradient stochastic dynamics). A detailed study of these scaling limits will be given elsewhere $[8,17]$.

Let us now briefly outline the structure of the paper.

In Section 2, we fix a Riemannian manifold $X$ as underlying space (the position space of the particles) and the space $\Gamma$ of all locally finite configurations in $X$. The restriction to the Riemannian manifold case is mainly motivated by the necessity to 
have constructive conditions for the existence of equilibrium states for interacting particle systems in $X$. Let us stress that all general statements of the paper (with minor changes) remain valid for much more general underlying spaces.

We next recall the definition of a Gibbs measure $\mu$ on $\Gamma$ which corresponds to a relative energy $E(x, \gamma)$ of the interaction between a particle $x$ and a configuration $\gamma$. About the measure $\mu$ we assume that it has correlation functions which satisfy the classical Ruelle bound. We also present some examples of a Gibbs measure corresponding to a pair potential $\phi$. It should be mentioned that, although in the examples we deal with a pair potential $\phi$, our general theory for existence of dynamics holds for a general relative energy $E(x, \gamma)$.

Next, in Section 3, under mild conditions on $E(x, \gamma)$, we prove that there exists a Hunt processes $\mathbf{M}$ on $\Gamma$ which is properly associated with the Dirichlet form of the Kawasaki dynamics. In particular, $\mathbf{M}$ is a conservative Markov process on $\Gamma$ with cadlag paths, and has $\mu$ as symmetrizing, hence invariant measure. We also characterize this process in terms of the corresponding martingale problem. Furthermore, we discuss the explicit form of the $L^{2}(\mu)$-generator of this process on the set of continuous bounded cylinder functions. In this section, we use the theory of Dirichlet forms [25], and in particular, some ideas and techniques developed in [18, 19, 26, 29].

In Section 4 we consider some examples of Kawasaki dynamics.

Finally, in Section 5, by analogy with the Kawasaki dynamics, we formulate conditions which guarantee the existence of an equilibrium Glauber (birth-and-death) dynamics in continuum (compare with $[15,18,28]$ ). We then outline the above mentioned scaling limits of the equilibrium Kawasaki dynamics.

We conclude this section with the following remarks. In a bounded domain, a Kawasaki dynamics can be described as a jump Markov process. However, in the infinite volume, this dynamics does not belong to this class, since in any time interval $[0, t]$, the dynamics has an infinite number of jumps. Note also that the set of symmetrizing measures of a given Kawasaki dynamics consists of all grand-canonical Gibbs measures corresponding to a given relative energy of interaction and any activity parameter $z>0$. This fact makes it especially interesting to study the hydrodynamic behavior of the Kawasaki dynamics, cf. [7, 32]. Finally, note a similarity between the Kawasaki dynamics and the diffusion dynamics of continuous particle systems, e.g. $[1,14,19,26]$. Namely, both types of dynanics have (at least heuristically) conserved particle numbers and the same set of symmetrizing measures. Therefore, just as in the diffusion case, it is natural to study the scaling limit of equilibrium fluctuations for the Kawasaki dynamics, which is the subject of [20]. 


\section{Gibbs measures on configuration spaces}

Let $X$ be a connected oriented $C^{\infty}$ manifold. We denote the Riemannian distance on $X$ by dist. Let $\mathcal{B}(X)$ denote the Borel $\sigma$-algebra on $X$ and $m$ the volume measure on $X$.

The configuration space $\Gamma:=\Gamma_{X}$ over $X$ is defined as the set of all subsets of $X$ which are locally finite:

$$
\Gamma:=\left\{\gamma \subset X:\left|\gamma_{\Lambda}\right|<\infty \text { for each compact } \Lambda \subset X\right\}
$$

where $|\cdot|$ denotes the cardinality of a set and $\gamma_{\Lambda}:=\gamma \cap \Lambda$. One can identify any $\gamma \in \Gamma$ with the positive Radon measure $\sum_{x \in \gamma} \varepsilon_{x} \in \mathcal{M}(X)$, where $\varepsilon_{x}$ is the Dirac measure with mass at $x, \sum_{x \in \varnothing} \varepsilon_{x}$ :=zero measure, and $\mathcal{M}(X)$ stands for the set of all positive Radon measures on $\mathcal{B}(X)$. The space $\Gamma$ can be endowed with the relative topology as a subset of the space $\mathcal{M}(X)$ with the vague topology, i.e., the weakest topology on $\Gamma$ with respect to which all maps

$$
\Gamma \ni \gamma \mapsto\langle f, \gamma\rangle:=\int_{X} f(x) \gamma(d x)=\sum_{x \in \gamma} f(x), \quad f \in C_{0}(X)
$$

are continuous. Here, $C_{0}(X)$ is the space of all continuous real-valued functions on $X$ with compact support. We shall denote the Borel $\sigma$-algebra on $\Gamma$ by $\mathcal{B}(\Gamma)$.

Now we proceed to consider Gibbs measures on $\Gamma$. For $\gamma \in \Gamma$ and $x \in X$, we consider a relative energy $E(x, \gamma)$ of interaction between a particle located at $x$ and the configuration $\gamma$. We suppose that the mapping $E$ is measurable and $E(x, \gamma) \in$ $(-\infty,+\infty]$.

A probability measure $\mu$ on $(\Gamma, \mathcal{B}(\Gamma))$ is called a (grand-canonical) Gibbs measure corresponding to activity $z>0$ and the relative energy $E$ if it satisfies the GeorgiiNguyen-Zessin identity ([27, Theorem 2], see also [22, Theorem 2.2.4]):

$$
\int_{\Gamma} \mu(d \gamma) \int_{X} \gamma(d x) F(x, \gamma)=\int_{\Gamma} \mu(d \gamma) \int_{X} z m(d x) \exp [-E(x, \gamma)] F(x, \gamma \cup x)
$$

for any measurable function $F: X \times \Gamma \rightarrow[0,+\infty]$. Let $\mathcal{G}(z, E)$ denote the set of all Gibbs measures corresponding to $z$ and $E$.

In particular, if $E(x, \gamma) \equiv 0$, then $(2.1)$ is the Mecke identity, which holds if and only if $\mu$ is the Poisson measure $\pi_{z}$ with intensity measure $z m(d x)$.

We assume that

$$
E(x, \gamma) \in \mathbb{R} \quad \text { for } m \otimes \mu \text {-a.e. }(x, \gamma) \in X \times \Gamma \text {. }
$$

Furthermore, we assume that, for any $n \in \mathbb{N}$, there exists a non-negative measurable symmetric function $k_{\mu}^{(n)}$ on $X^{n}$ such that, for any measurable symmetric function $f^{(n)}$ : 


$$
\begin{aligned}
& X^{n} \rightarrow[0, \infty] \\
& \qquad \int_{\Gamma_{\left\{x_{1}, \ldots, x_{n}\right\} \subset \gamma} \sum^{(n)}\left(x_{1}, \ldots, x_{n}\right) \mu(d \gamma)} \\
& \quad=\frac{1}{n !} \int_{X^{n}} f^{(n)}\left(x_{1}, \ldots, x_{n}\right) k_{\mu}^{(n)}\left(x_{1}, \ldots, x_{n}\right) m\left(d x_{1}\right) \cdots m\left(d x_{n}\right),
\end{aligned}
$$

and

$$
\forall\left(x_{1}, \ldots, x_{n}\right) \in X^{n}: \quad k_{\mu}^{(n)}\left(x_{1}, \ldots, x_{n}\right) \leq \xi^{n},
$$

where $\xi>0$ is independent of $n$. The functions $k_{\mu}^{(n)}, n \in \mathbb{N}$, are called the correlation functions of the measure $\mu$, while (2.4) is called the Ruelle bound.

Notice that any probability measure $\mu$ on $(\Gamma, \mathcal{B}(\Gamma))$ satisfyng the Ruelle bound has all local moments finite, i.e.,

$$
\int_{\Gamma}\langle f, \gamma\rangle^{n} \mu(d \gamma)<\infty, \quad f \in C_{0}(X), f \geq 0, n \in \mathbb{N}
$$

Let us give examples of a Gibbs measure corresponding to a pair potential $\phi$ and satisfying the above assumptions.

Let $\phi: X^{2} \rightarrow(-\infty,+\infty]$ be a symmetric measurable function such that $\phi(x, y) \in \mathbb{R}$ for any $x, y \in X, x \neq y$. For each $x \in X$ and $\gamma \in \Gamma$, we define

$$
E(x, \gamma):= \begin{cases}\sum_{y \in \gamma} \phi(x, y), & \text { if } \sum_{y \in \gamma}|\phi(x, y)|<\infty \\ +\infty, & \text { otherwise }\end{cases}
$$

Let us formulate some conditions on the pair potential $\phi$.

(S) (Stability) There exists $B \geq 0$ such that, for any $\gamma \in \Gamma,|\gamma|<\infty$,

$$
\sum_{\{x, y\} \subset \gamma} \phi(x, y) \geq-B|\gamma|
$$

(I) (Integrability) We have

$$
C:=\sup _{x \in X} \int_{X}|\exp [-\phi(x, y)]-1| m(d y)<\infty
$$

(F) (Finite range) There exists $R>0$ such that

$$
\phi(x, y)=0 \quad \text { if } \operatorname{dist}(x, y) \geq R
$$

Note that if $\phi$ satisfies $(\mathrm{F})$, then $E(x, \gamma) \in \mathbb{R}$ for any $\gamma \in \Gamma$ and $x \in X \backslash \gamma$. 
Theorem $2.1([16,22,23])$ 1) Let (S), (I), and (F) hold, and let $z>0$ be such that

$$
z<\frac{1}{2 e}\left(e^{2 B} C\right)^{-1},
$$

where $B$ and $C$ are as in $(\mathrm{S})$ and $(\mathrm{I})$, respectively. Then there exists a Gibbs measure $\mu \in \mathcal{G}(z, E)$ whose correlation functions $k_{\mu}^{(n)}$ exist and satisfy the Ruelle bound.

2) Let $\phi$ be a non-negative potential which fulfills (I) and (F). Then for each $z>0$, there exists a Gibbs measure $\mu \in \mathcal{G}(z, E)$ whose correlation functions $k_{\mu}^{(n)}$ exist and satisfy the Ruelle bound.

Assume now that $X=\mathbb{R}^{d}, d \in \mathbb{N}$, and assume that $\phi$ is translation invariant, i.e., $\phi(x, y)=\tilde{\phi}(x-y)$, where $\tilde{\phi}: \mathbb{R} \rightarrow(-\infty, \infty]$ is such that $\tilde{\phi}(x) \in \mathbb{R}$ for $x \neq 0$ and $\tilde{\phi}(-x)=\tilde{\phi}(x)$ for all $x \in \mathbb{R}^{d}$. In this case, the conditions on $z$ and $\phi$ can be significantly weakened. First, we note that the condition (I) now looks as follows:

$$
C:=\int_{\mathbb{R}^{d}}|\exp [-\tilde{\phi}(x)]-1| m(d x)<\infty .
$$

For the notion of a superstable, lower regular potential and the notion of a tempered Gibbs measure, appearing in the following theorem, see [31].

Theorem $2.2([30,31])$ Assume that $X=\mathbb{R}^{d}$ and $\phi$ is translation invariant.

1) Let (S) and (I) hold and let $z>0$ be such that

$$
z<\frac{1}{e}\left(e^{2 B} C\right)^{-1},
$$

where $B$ and $C$ are as in $(\mathrm{S})$ and $(\mathrm{I})$, respectively. Then there exists a Gibbs measure $\mu \in \mathcal{G}(z, E)$ whose correlation functions exist and satisfy the Ruelle bound.

2) Let $\phi$ be a non-negative potential which fulfills (I). Then, for each $z>0$, there exists a Gibbs measure $\mu \in \mathcal{G}(z, E)$ whose correlation functions exist and satisfy the Ruelle bound.

3) Let $\phi$ satisfy (I) and additionally let $\phi$ be a superstable, lower regular potential. Then the set $\mathcal{G}_{\text {temp }}(z, E)$ of all tempered Gibbs measures is non-empty and each measure from $\mathcal{G}_{\text {temp }}(z, E)$ has correlation functions which satisfy the Ruelle bound.

We also have the following lemma, which follows from (the proof of) [19, Lemma 3.1].

Lemma 2.1 Let $X=\mathbb{R}^{d}$ and let $\phi, z$, and $\mu \in \mathcal{G}(z, E)$ be as in one of the statements of Theorem 2.2. Assume additionally that there exists $r>0$ such that

$$
\sup _{x \in B(r)^{c}} \tilde{\phi}(x)<\infty,
$$

where $B(r)$ denotes the ball in $\mathbb{R}^{d}$ of radius $r$ centered at the origin. Then $(2.2)$ holds. 


\section{Existence results}

In what follows, we shall consider a Gibbs measure $\mu \in \mathcal{G}(z, E)$ as in Section 2, i.e., a probability measure $\mu$ on $(\Gamma, \mathcal{B}(\Gamma))$ which satisfies $(2.1)-(2.4)$. We introduce the set $\mathcal{F} C_{\mathrm{b}}\left(C_{0}(X), \Gamma\right)$ of all functions of the form

$$
\Gamma \ni \gamma \mapsto F(\gamma)=g_{F}\left(\left\langle\varphi_{1}, \gamma\right\rangle, \ldots,\left\langle\varphi_{N}, \gamma\right\rangle\right)
$$

where $N \in \mathbb{N}, \varphi_{1}, \ldots, \varphi_{N} \in C_{0}(X)$ and $g_{F} \in C_{\mathrm{b}}\left(\mathbb{R}^{N}\right)$, where $C_{\mathrm{b}}\left(\mathbb{R}^{N}\right)$ denotes the set of all continuous bounded functions on $\mathbb{R}^{N}$.

We consider a measurable mapping

$$
X \times X \times \Gamma \ni(x, y, \gamma) \mapsto c(x, y, \gamma) \in[0, \infty)
$$

We assume that, for each compact $\Lambda \subset X$,

$$
\int_{\Gamma} \mu(d \gamma) \int_{X} \gamma(d x) \int_{X} m(d y) c(x, y, \gamma)\left(\mathbf{1}_{\Lambda}(x)+\mathbf{1}_{\Lambda}(y)\right)<\infty
$$

where $\mathbf{1}_{\Lambda}$ denotes the indicator of $\Lambda$.

For each function $F: \Gamma \rightarrow \mathbb{R}, \gamma \in \Gamma$, and $x, y \in X$, we recall the notation (1.2). Then we define a bilinear form

$$
\mathcal{E}(F, G):=\int_{\Gamma} \mu(d \gamma) \int_{X} \gamma(d x) \int_{X} z m(d y) c(x, y, \gamma)\left(D_{x y}^{-+} F\right)(\gamma)\left(D_{x y}^{-+} G\right)(\gamma)
$$

where $F, G \in \mathcal{F} C_{\mathrm{b}}\left(C_{0}(X), \Gamma\right)$. Below we shall show that $\mathcal{E}$ corresponds to a Kawasaki dynamics.

We note that, for any $F \in \mathcal{F} C_{\mathrm{b}}\left(C_{0}(X), \Gamma\right)$, there exist a compact $\Lambda \subset X$ and $C_{1}>0$ such that

$$
\left|\left(D_{x y}^{-+} F\right)(\gamma)\right| \leq C_{1}\left(\mathbf{1}_{\Lambda}(x)+\mathbf{1}_{\Lambda}(y)\right), \quad \gamma \in \Gamma, x, y \in X .
$$

Therefore, by (3.1), the right hand sides of formula (3.2) is well-defined and finite.

Lemma 3.1 We have $\mathcal{E}(F, G)=0$ for all $F, G \in \mathcal{F} C_{\mathrm{b}}\left(C_{0}(X), \Gamma\right)$ such that $F=0$

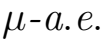

Proof. It suffices to show that, for $F \in \mathcal{F} C_{\mathrm{b}}\left(C_{0}(X), \Gamma\right), F=0 \mu$-a.e., we have $\left(D_{x, y}^{-+} F\right)(\gamma)=0 \tilde{\mu}$-a.e., where $\tilde{\mu}$ is the measure on $X \times X \times \Gamma$ defined by

$$
\tilde{\mu}(d x, d y, d \gamma):=\gamma(d x) z m(d y) \mu(d \gamma) .
$$

Let $\Lambda$ be a compact subset of $X$. We have:

$$
\int_{\Gamma} \mu(d \gamma) \int_{\Lambda} \gamma(d x) \int_{\Lambda} z m(d y)|F(\gamma)|=\int_{\Gamma} \mu(d \gamma)|F(\gamma)| \int_{\Lambda} \gamma(d x) \int_{\Lambda} z m(d y)=0
$$


which implies that $F(\gamma)=0 \tilde{\mu}$-a.e. Next, by $(2.1)$ and $(2.2)$,

$$
\begin{aligned}
\int_{\Gamma} \mu(d \gamma) & \int_{\Lambda} \gamma(d x) \int_{\Lambda} z m(d y)|F(\gamma \backslash x \cup y)| \\
= & \int_{\Gamma} \mu(d \gamma)|F(\gamma)| \int_{\Lambda} \gamma(d x) \int_{\Lambda} z m(d y) \exp [-E(y, \gamma)+E(x, \gamma \backslash x \cup y)]
\end{aligned}
$$

Since $F$ is bounded, by (2.5), the integrals in (3.4) are finite. Therefore,

$$
|F(\gamma)| \exp [-E(y, \gamma)+E(x, \gamma \backslash x \cup y)]<\infty \quad \text { for } \tilde{\mu} \text {-a.e. }(x, y, \gamma) \in X \times X \times \Gamma \text {. }
$$

Since $F=0 \mu$-a.e., by (3.4) and (3.5), $F(\gamma \backslash x \cup y)=0 \tilde{\mu}$-a.e.

Thus, $\left(\mathcal{E}, \mathcal{F} C_{\mathrm{b}}\left(C_{0}(X), \Gamma\right)\right)$ is a well-defined bilinear form on $L^{2}(\Gamma, \mu)$.

Lemma 3.2 The bilinear form $\left(\mathcal{E}, \mathcal{F} C_{\mathrm{b}}\left(C_{0}(X), \Gamma\right)\right)$ is closable on $L^{2}(\Gamma, \mu)$ and its closure will be denoted by $(\mathcal{E}, D(\mathcal{E}))$.

Proof. Let $\left(F_{n}\right)_{n=1}^{\infty}$ be a sequence in $\mathcal{F} C_{\mathrm{b}}\left(C_{0}(X), \Gamma\right)$ such that $\left\|F_{n}\right\|_{L^{2}(\mu)} \rightarrow 0$ as $n \rightarrow \infty$ and

$$
\mathcal{E}\left(F_{n}-F_{k}\right) \rightarrow 0 \quad \text { as } n, k \rightarrow \infty .
$$

Here and below, $\mathcal{E}(F)$ stays for $\mathcal{E}(F, F)$. To prove the closability of $\mathcal{E}$ it suffices to show that there exists a subsequence $\left(F_{n_{k}}\right)_{k=1}^{\infty}$ such that $\mathcal{E}\left(F_{n_{k}}\right) \rightarrow 0$ as $k \rightarrow \infty$.

Let $\Lambda$ be a compact subset of $X$. By (2.5), we have

$$
\int_{\Gamma} \mu(d \gamma) \int_{\Lambda} \gamma(d x)\left|F_{n}(\gamma)\right| \leq\left\|F_{n}\right\|_{L^{2}(\mu)}\left(\int_{\Gamma}\left\langle\mathbf{1}_{\Lambda}, \gamma\right\rangle^{2} \mu(d \gamma)\right)^{1 / 2} \rightarrow 0 \quad \text { as } n \rightarrow \infty
$$

Therefore, there exists a subsequence of $\left(F_{n}\right)_{n=1}^{\infty}$, denoted by $\left(F_{n}^{(1)}\right)_{n=1}^{\infty}$, such that $F_{n}^{(1)}(\gamma) \rightarrow 0$ for $\gamma(d x) \mu(d \gamma)$-a.e. $(x, \gamma) \in \Lambda \times \Gamma$. Hence, there exists a subsequence $\left(F_{n}^{(2)}\right)_{n=1}^{\infty}$ of $\left(F_{n}^{(1)}\right)_{n=1}^{\infty}$ such that $F_{n}^{(2)}(\gamma) \rightarrow 0$ for $\gamma(d x) \mu(d \gamma)$-a.e. $(x, \gamma) \in X \times \Gamma$.

Next, analogously to (3.4),

$$
\begin{aligned}
\int_{\Gamma} \mu(d \gamma) \int_{\Lambda} \gamma(d x) \int_{\Lambda} z m(d y) \exp [-E(y, \gamma)+E(x, \gamma \backslash x \cup y)]\left|F_{n}^{(2)}(\gamma \backslash x \cup y)\right| \\
\quad=\int_{\Gamma} \mu(d \gamma) \int_{\Lambda} z m(d x) \int_{\Lambda} \gamma(d y)\left|F_{n}(\gamma)\right| \\
\quad \leq\left\|F_{n}^{(2)}\right\|_{L^{2}(\mu)} z m(\Lambda)\left(\int_{\Gamma}\left\langle\mathbf{1}_{\Lambda}, \gamma\right\rangle^{2} \mu(d \gamma)\right)^{1 / 2} \rightarrow 0 \quad \text { as } n \rightarrow \infty .
\end{aligned}
$$

By virtue of (2.2),

$$
\exp [-E(y, \gamma)+E(x, \gamma \backslash x \cup y)] \in(0,+\infty] \text { for } \tilde{\mu} \text {-a.e. }(x, y, \gamma) \in X \times X \times \Gamma \text {. }
$$


Therefore, there exists a subsequence $\left(F_{n}^{(3)}\right)_{n=1}^{\infty}$ of $\left(F_{n}^{(2)}\right)_{n=1}^{\infty}$ such that $F_{n}^{(3)}(\gamma \backslash x \cup y) \rightarrow 0$ for $\tilde{\mu}$-a.e. $(x, y, \gamma) \in X \times X \times \Gamma$, where the measure $\tilde{\mu}$ is defined by (3.3).

Thus,

$$
\left(D_{x y}^{-+} F_{n}^{(3)}\right)(\gamma) \rightarrow 0 \quad \text { as } n \rightarrow \infty \text { for } \tilde{\mu} \text {-a.e. }(x, y, \gamma) \in X \times X \times \Gamma .
$$

Now, by (3.7) and Fatou's lemma

$$
\begin{aligned}
\mathcal{E}\left(F_{n}^{(3)}\right) & =\int c(x, y, \gamma)\left(D_{x y}^{-+} F_{n}^{(3)}\right)(\gamma)^{2} \tilde{\mu}(d x, d y, d \gamma) \\
& =\int c(x, y, \gamma)\left(\left(D_{x y}^{-+} F_{n}^{(3)}\right)(\gamma)-\lim _{m \rightarrow \infty}\left(D_{x y}^{-+} F_{m}^{(3)}\right)(\gamma)\right)^{2} \tilde{\mu}(d x, d y, d \gamma) \\
& \leq \liminf _{m \rightarrow \infty} \int c(x, y, \gamma)\left(\left(D_{x y}^{-+} F_{n}^{(3)}\right)(\gamma)-\left(D_{x y}^{-+} F_{m}^{(3)}\right)(\gamma)\right)^{2} \tilde{\mu}(d x, d y, d \gamma) \\
& =\liminf _{m \rightarrow \infty} \mathcal{E}\left(F_{n}^{(3)}-F_{m}^{(3)}\right),
\end{aligned}
$$

which by (3.6) can be made arbitrarily small for $n$ large enough.

For the notion of a Dirichlet form, appearing in the following lemma, we refer to e.g. [25, Chap. I, Sect. 4].

Lemma $3.3\left(\mathcal{E}, D(\mathcal{E})\right.$ is a Dirichlet form on $L^{2}(\Gamma, \mu)$.

Proof. On $D(\mathcal{E})$ we consider the norm $\|F\|_{D(\mathcal{E})}:=\left(\|F\|_{L^{2}(\mu)}^{2}+\mathcal{E}(F)\right)^{1 / 2}, F \in D(\mathcal{E})$. For any $F, G \in \mathcal{F} C_{\mathrm{b}}\left(C_{0}(X), \Gamma\right)$, we define

$$
S(F, G)(x, y, \gamma):=c(x, y, \gamma)\left(D_{x y}^{-+} F\right)(\gamma)\left(D_{x y}^{-+} G\right)(\gamma), \quad x, y \in X, \gamma \in \Gamma .
$$

Using the Cauchy inequality, we conclude that $S$ extends to a bilinear continuous map from $\left(D(\mathcal{E}),\|\cdot\|_{D(\mathcal{E})}\right) \times\left(D(\mathcal{E}),\|\cdot\|_{D(\mathcal{E})}\right)$ into $L^{1}(X \times X \times \Gamma, \tilde{\mu})$. Let $F \in D(\mathcal{E})$ and consider any sequence $\left(F_{n}\right)_{n=1}^{\infty}$ in $\mathcal{F} C_{\mathrm{b}}\left(C_{0}(X), \Gamma\right)$ such that $F_{n} \rightarrow F$ in $\left(D(\mathcal{E}),\|\cdot\|_{D(\mathcal{E})}\right)$. In particular, $F_{n} \rightarrow F$ in $L^{2}(\mu)$. Then, analogously to the proof of Lemma 3.2, for some subsequence $\left(F_{n_{k}}\right)_{k=1}^{\infty}$, we get

$$
\left(D_{x y}^{-+} F_{n_{k}}\right)(\gamma) \rightarrow\left(D_{x y}^{-+} F\right)(\gamma) \quad \text { for } \tilde{\mu} \text {-a.e. }(x, y, \gamma) \in X \times X \times \Gamma \text {. }
$$

Therefore, for any $F, G \in D(\mathcal{E})$,

$$
S(F, G)(x, y, \gamma):=c(x, y, \gamma)\left(D_{x y}^{-+} F\right)(\gamma)\left(D_{x y}^{-+} G\right)(\gamma) \quad \text { for } \tilde{\mu} \text {-a.e. }(x, y, \gamma) \in X \times X \times \Gamma
$$

and

$$
\mathcal{E}(F, G)=\int S(F, G)(x, y, \gamma) \tilde{\mu}(d x, d y, d \gamma)
$$


Define $\mathbb{R} \ni x \mapsto g(x):=(0 \vee x) \wedge 1$. We again fix any $F \in D(\mathcal{E})$ and let $\left(F_{n}\right)_{n=1}^{\infty}$ be a sequence of functions from $\mathcal{F} C_{\mathrm{b}}\left(C_{0}(X), \Gamma\right)$ such that $F_{n} \rightarrow F$ in $\left(D(\mathcal{E}),\|\cdot\|_{D(\mathcal{E})}\right)$. Consider the sequence $\left(g\left(F_{n}\right)\right)_{n \in \mathbb{N}}$. We evidently have: $g\left(F_{n}\right) \in \mathcal{F} C_{\mathrm{b}}\left(C_{0}(X), \Gamma\right)$ for each $n \in \mathbb{N}$ and, by the dominated convergence theorem, $g\left(F_{n}\right) \rightarrow g(F)$ as $n \rightarrow \infty$ in $L^{2}(\mu)$. Next, by the above argument, we have, for some subsequence $\left(F_{n_{k}}\right)_{k=1}^{\infty}$, $\left(D_{x y}^{-+} g\left(F_{n_{k}}\right)\right)(\gamma) \rightarrow\left(D_{x y}^{-+} g(F)\right)(\gamma)$ as $n \rightarrow \infty$ for $\tilde{\mu}$-a.e. $(x, y, \gamma)$.

For any $x, y \in \mathbb{R}$, we evidently have

$$
|g(x)-g(y)| \leq|x-y|
$$

Therefore, the sequence $c(x, y, \gamma)^{1 / 2}\left(D_{x y}^{-+} g\left(F_{n}\right)\right)(\gamma), n \in \mathbb{N}$, is $\tilde{\mu}$-uniformly squareintegrable, since so is the sequence $c(x, y, \gamma)^{1 / 2}\left(D_{x y}^{-+} F_{n}\right)(\gamma), n \in \mathbb{N}$. Hence

$$
c(x, y, \gamma)^{1 / 2}\left(D_{x y}^{-+} g\left(F_{n_{k}}\right)\right)(\gamma) \rightarrow c(x, y, \gamma)^{1 / 2}\left(D_{x y}^{-+} g(F)\right)(\gamma) \quad \text { as } k \rightarrow \infty \text { in } L^{2}(\tilde{\mu}) .
$$

By (3.8) and (3.9), this yields: $g(F) \in D(\mathcal{E})$.

Finally, by $(3.8)-(3.10), \mathcal{E}(g(F)) \leq \mathcal{E}(F)$, which means that $(\mathcal{E}, D(\mathcal{E})))$ is a Dirichlet form.

We shall now need the bigger space $\ddot{\Gamma}$ consisting of all $\mathbb{Z}_{+} \cup\{\infty\}$-valued Radon measures on $X$ (which is Polish, see e.g. [21]). Since $\Gamma \subset \ddot{\Gamma}$ and $\mathcal{B}(\ddot{\Gamma}) \cap \Gamma=\mathcal{B}(\Gamma)$, we can consider $\mu$ as a measure on $(\ddot{\Gamma}, \mathcal{B}(\ddot{\Gamma}))$ and correspondingly $(\mathcal{E}, D(\mathcal{E}))$ as a Dirichlet form on $L^{2}(\ddot{\Gamma}, \mu)$.

For the notion of a quasi-regular Dirichlet form, appearing in the following lemma, we refer to [25, Chap. IV, Sect. 3].

Lemma $3.4(\mathcal{E}, D(\mathcal{E}))$ is a quasi-regular Dirichlet form on $L^{2}(\ddot{\Gamma}, \mu)$.

Proof. Analogously to [26, Proposition 4.1], it suffices to show that there exists a bounded, complete metric $\rho$ on $\ddot{\Gamma}$ generating the vague topology such that, for all $\gamma_{0} \in \ddot{\Gamma}, \rho\left(\cdot, \gamma_{0}\right) \in D(\mathcal{E})$ and

$$
\int_{X} \gamma(d x) \int_{X} z m(d y) S\left(\rho\left(\cdot, \gamma_{0}\right)\right)(x, y, \gamma) \leq \eta(\gamma) \quad \mu \text {-a.e. }
$$

for some $\eta \in L^{1}(\ddot{\Gamma}, \mu)$ (independent of $\left.\gamma_{0}\right)$. Here, $S(F):=S(F, F)$. The proof below is a modification of the proof of [26, Proposition 4.8] and the proof of [18, Proposition 3.2].

Fix any $x_{0} \in X$, let $B(r)$ denote the open ball in $X$ of radius $r>0$ centered at $x_{0}$. For each $k \in \mathbb{N}$, we define

$$
g_{k}(x):=\frac{2}{3}\left(\frac{1}{2}-\operatorname{dist}(x, B(k)) \wedge \frac{1}{2}\right), \quad x \in X,
$$


where $\operatorname{dist}(x, B(k))$ denotes the distance from the point $x$ to the ball $B(k)$. Next, we set

$$
\phi_{k}(x):=3 g_{k}(x), \quad x \in X, k \in \mathbb{N} .
$$

Let $\zeta$ be a function in $C_{\mathrm{b}}^{1}(\mathbb{R})$ such that $0 \leq \zeta \leq 1$ on $[0, \infty), \zeta(t)=t$ on $[-1 / 2,1 / 2]$, $\zeta^{\prime} \in[0,1]$ on $[0, \infty)$. For any fixed $\gamma_{0} \in \ddot{\Gamma}$ and for any $k, n \in \mathbb{N}$, (the restriction to $\Gamma$ of) the function

$$
\zeta\left(\sup _{j \leq n}\left|\left\langle\phi_{k} g_{j}, \cdot\right\rangle-\left\langle\phi_{k} g_{j}, \gamma_{0}\right\rangle\right|\right)
$$

belongs to $\mathcal{F} C_{\mathrm{b}}\left(C_{0}(X), \Gamma\right)$ (note that $\left\langle\phi_{k} g_{j}, \gamma_{0}\right\rangle$ is a constant). Furthermore, taking into account that $\zeta^{\prime} \in[0,1]$ on $[0, \infty)$, we get from the mean value theorem, for each $\gamma \in \Gamma, x \in \gamma$, and $x \in X \backslash \gamma$,

$$
\begin{aligned}
S\left(\zeta\left(\sup _{j \leq n}\left|\left\langle\phi_{k} g_{j}, \cdot\right\rangle-\left\langle\phi_{k} g_{j}, \gamma_{0}\right\rangle\right|\right)\right)(x, y, \gamma) \\
\leq c(x, y, \gamma)\left(\sup _{j \leq n}\left|\left\langle\phi_{k} g_{j}, \gamma\right\rangle-\left\langle\phi_{k} g_{j}, \gamma_{0}\right\rangle-\left(\phi_{k} g_{j}\right)(x)+\left(\phi_{k} g_{j}\right)(y)\right|\right. \\
\left.\quad-\sup _{j \leq n}\left|\left\langle\phi_{k} g_{j}, \gamma\right\rangle-\left\langle\phi_{k} g_{j}, \gamma_{0}\right\rangle\right|\right)^{2} \\
\leq c(x, y, \gamma) \sup _{j \leq n}\left|-\left(\phi_{k} g_{j}\right)(x)+\left(\phi_{k} g_{j}\right)(y)\right|^{2} \\
\leq 2 c(x, y, \gamma)\left(\sup _{j \leq n}\left(\phi_{k} g_{j}\right)(x)^{2}+\sup _{j \leq n}\left(\phi_{k} g_{j}\right)(y)^{2}\right) \\
\leq 2 c(x, y, \gamma)\left(\mathbf{1}_{B(k+1 / 2)}(x)+\mathbf{1}_{B(k+1 / 2)}(y)\right) .
\end{aligned}
$$

For each $k \in \mathbb{N}$, we define

$$
F_{k}\left(\gamma, \gamma_{0}\right):=\zeta\left(\sup _{j \in \mathbb{N}}\left|\left\langle\phi_{k} g_{j}, \gamma\right\rangle-\left\langle\phi_{k} g_{j}, \gamma_{0}\right\rangle\right|\right), \quad \gamma, \gamma_{0} \in \ddot{\Gamma} .
$$

Then, for a fixed $\gamma_{0} \in \ddot{\Gamma}$,

$$
\zeta\left(\sup _{j \leq n}\left|\left\langle\phi_{k} g_{j}, \gamma\right\rangle-\left\langle\phi_{k} g_{j}, \gamma_{0}\right\rangle\right|\right) \rightarrow F_{k}\left(\gamma, \gamma_{0}\right)
$$

as $n \rightarrow \infty$ for each $\gamma \in \ddot{\Gamma}$ and in $L^{2}(\mu)$. Hence, by (3.11) and the Banach-Alaoglu and the Banach-Saks theorems (see e.g. [25, Appendix A.2]), $F_{k}\left(\cdot, \gamma_{0}\right) \in D(\mathcal{E})$ and

$$
S\left(F_{k}\left(\cdot, \gamma_{0}\right)\right)(x, y, \gamma) \leq 2 c(x, y, \gamma)\left(\mathbf{1}_{B(k+1 / 2)}(x)+\mathbf{1}_{B(k+1 / 2)}(y)\right) \quad \tilde{\mu} \text {-a.e. }
$$

Define

$c_{k}:=\left(1+2 \int c(x, y, \gamma)\left(\mathbf{1}_{B(k+1 / 2)}(x)+\mathbf{1}_{B(k+1 / 2)}(y)\right) \tilde{\mu}(d x, d y, d \gamma)\right)^{-1 / 2} 2^{-k / 2}, \quad k \in \mathbb{N}$, 
which are finite positive numbers by (3.1), and furthermore, $c_{k} \rightarrow 0$ as $k \rightarrow \infty$.

We define

$$
\rho\left(\gamma_{1}, \gamma_{2}\right):=\sup _{k \in \mathbb{N}}\left(c_{k} F_{k}\left(\gamma_{1}, \gamma_{2}\right)\right), \quad \gamma_{1}, \gamma_{2} \in \ddot{\Gamma} .
$$

By [26, Theorem 3.6], $\rho$ is a bounded, complete metric on $\ddot{\Gamma}$ generating the vague topology.

Analogously to the above, we now conclude that, for any fixed $\gamma_{0} \in \ddot{\Gamma}, \rho\left(\cdot, \gamma_{0}\right) \in$ $D(\mathcal{E})$ and

$$
\int_{X} \gamma(d x) \int_{X} z m(d y) S\left(\rho\left(\cdot, \gamma_{0}\right)\right)(x, y, \gamma) \leq \eta(\gamma) \quad \mu \text {-a.e., }
$$

where

$$
\eta(\gamma):=2 \sup _{k \in \mathbb{N}}\left(c_{k}^{2} \int_{X} \gamma(d x) \int_{X} z m(d y) c(x, y, \gamma)\left(\mathbf{1}_{B(k+1 / 2)}(x)+\mathbf{1}_{B(k+1 / 2)}(y)\right)\right) .
$$

Finally,

$$
\begin{aligned}
\int_{\Gamma} \eta(\gamma) \mu(d \gamma) & \leq 2 \sum_{k=1}^{\infty} c_{k}^{2} \int c(x, y, \gamma)\left(\mathbf{1}_{B(k+1 / 2)}(x)+\mathbf{1}_{B(k+1 / 2)}(y)\right) \tilde{\mu}(d x, d y, d \gamma) \\
& \leq \sum_{k=1}^{\infty} 2^{-k}=1 .
\end{aligned}
$$

Thus, the lemma is proved.

For the notion of an exceptional set, appearing in the next proposition, we refer e.g. to [25, Chap. III, Sect. 2].

Lemma 3.5 The set $\ddot{\Gamma} \backslash \Gamma$ is $\mathcal{E}$-exceptional.

Proof. We modify the proof of [29, Proposition 1 and Corollary 1] and the proof of [18, Proposition 3.3] according to our situation.

It suffices to prove the result locally, i.e., to show that, for any fixed $a \in X$, there exists a closed set $B_{a}$ that is the closure of an open neighborhood of $a$ and such that the set

$$
N_{a}:=\left\{\gamma \in \ddot{\Gamma}: \sup _{x \in B_{a}} \gamma(\{x\}) \geq 2\right\}
$$

is $\mathcal{E}$-exceptional. By [29, Lemma 1], we need to prove that there exists a sequence $u_{n} \in D(\mathcal{E}), n \in \mathbb{N}$, such that each $u_{n}$ is a continuous function on $\ddot{\Gamma}, u_{n} \rightarrow \mathbf{1}_{N_{a}}$ pointwise as $n \rightarrow \infty$, and $\sup _{n \in \mathbb{N}} \mathcal{E}\left(u_{n}\right)<\infty$.

So, we fix $a \in X$. There exists an open neighborhood $\tilde{B}_{a}$ of $a$ which is diffeomorphic to the open cube $(-3,+3)^{d}$ in $\mathbb{R}^{d}$. We fix the corresponding coordinate system in $\tilde{B}_{a}$ and we set $B_{a}:=[-1,1]^{d}$. 
Let $f \in C_{0}(\mathbb{R})$ be such that $\mathbf{1}_{[0,1]} \leq f \leq \mathbf{1}_{[-1 / 2,3 / 2)}$. For any $n \in \mathbb{N}$ and $i=$ $\left(i_{1}, \ldots, i_{d}\right) \in \mathcal{A}_{n}:=\mathbb{Z}^{d} \cap[-n, n]^{d}$, we define a function $f_{i}^{(n)} \in C_{0}(X)$ by

$$
f_{i}^{(n)}(x):= \begin{cases}\prod_{k=1}^{d} f\left(n x_{k}-i_{k}\right), & x \in \tilde{B}_{a}, \\ 0, & \text { otherwise }\end{cases}
$$

Let also

$$
I_{i}^{(n)}(x):= \begin{cases}\prod_{k=1}^{d} \mathbf{1}_{[-1 / 2,3 / 2)}\left(n x_{k}-i_{k}\right), & x \in \tilde{B}_{a}, \\ 0, & \text { otherwise }\end{cases}
$$

and note that $f_{i}^{(n)} \leq I_{i}^{(n)}$.

Let $\psi \in C_{\mathrm{b}}^{1}(\mathbb{R})$ be such that $\mathbf{1}_{[2, \infty)} \leq \psi \leq \mathbf{1}_{[1, \infty)}$ and $0 \leq \psi^{\prime} \leq 2 \mathbf{1}_{(1, \infty)}$. We define continuous functions

$$
\ddot{\Gamma} \ni \gamma \mapsto u_{n}(\gamma):=\psi\left(\sup _{i \in \mathcal{A}_{n}}\left\langle f_{i}^{(n)}, \gamma\right\rangle\right), \quad n \in \mathbb{N},
$$

whose restriction to $\Gamma$ belongs to $\mathcal{F} C_{\mathrm{b}}\left(C_{0}(X), \Gamma\right)$. Evidently, $u_{n} \rightarrow \mathbf{1}_{N_{a}}$ pointwise as $n \rightarrow \infty$.

By the mean value theorem, we have, for each $\gamma \in \Gamma, x \in \gamma, y \in X \backslash \gamma$, and for some point $T_{n}(x, y, \gamma)$ between $\sup _{i \in \mathcal{A}_{n}}\left\langle f_{i}^{(n)}, \gamma \backslash x \cup y\right\rangle$ and $\sup _{i \in \mathcal{A}_{n}}\left\langle f_{i}^{(n)}, \gamma\right\rangle$ :

$$
\begin{gathered}
S\left(u_{n}\right)(x, y, \gamma)=c(x, y, \gamma) \psi^{\prime}\left(T_{n}(x, y, \gamma)\right)^{2}\left(\sup _{i \in \mathcal{A}_{n}}\left\langle f_{i}^{(n)}, \gamma \backslash x \cup y\right\rangle-\sup _{i \in \mathcal{A}_{n}}\left\langle f_{i}^{(n)}, \gamma\right\rangle\right)^{2} \\
\leq c(x, y, \gamma) \psi^{\prime}\left(T_{n}(x, y, \gamma)\right)^{2} \sup _{i \in \mathcal{A}_{n}}\left|\left\langle f_{i}^{(n)}, \gamma \backslash x \cup y\right\rangle-\left\langle f_{i}^{(n)}, \gamma\right\rangle\right|^{2} \\
\leq 2 c(x, y, \gamma) \psi^{\prime}\left(T_{n}(x, y, \gamma)\right)^{2}\left(\sup _{i \in \mathcal{A}_{n}} f_{i}^{(n)}(x)^{2}+\sup _{i \in \mathcal{A}_{n}} f_{i}^{(n)}(y)^{2}\right) \\
\leq 8 c(x, y, \gamma)\left(\mathbf{1}_{\tilde{B}_{a}}(x)+\mathbf{1}_{\tilde{B}_{a}}(y)\right) .
\end{gathered}
$$

By (3.1) and (3.12), we conclude that

$$
\sup _{n \in \mathbb{N}} \mathcal{E}\left(u_{n}\right)<\infty,
$$

which implies the lemma.

We now have the main result of this paper.

Theorem 3.1 There exists a conservative Hunt process

$$
\mathbf{M}=\left(\boldsymbol{\Omega}, \mathbf{F},\left(\mathbf{F}_{t}\right)_{t \geq 0},\left(\boldsymbol{\Theta}_{t}\right)_{t \geq 0},(\mathbf{X}(t))_{t \geq 0},\left(\mathbf{P}_{\gamma}\right)_{\gamma \in \Gamma}\right)
$$


on $\Gamma$ (see e.g. $[25$, p. 92]) which is properly associated with $(\mathcal{E}, D(\mathcal{E}))$, i.e., for all $(\mu$ versions of ) $F \in L^{2}(\Gamma, \mu)$ and all $t>0$ the function

$$
\Gamma \ni \gamma \mapsto p_{t} F(\gamma):=\int_{\mathbf{\Omega}} F(\mathbf{X}(t)) d \mathbf{P}_{\gamma}
$$

is an $\mathcal{E}$-quasi-continuous version of $\exp (-t H) F$, where $(H, D(H))$ is the generator of $(\mathcal{E}, D(\mathcal{E})) . \quad \mathbf{M}$ is up to $\mu$-equivalence unique (cf. [25, Chap. IV, Sect. 6]). In particular, $\mathbf{M}$ is $\mu$-symmetric (i.e., $\int G p_{t} F d \mu=\int F p_{t} G d \mu$ for all $F, G: \Gamma \rightarrow \mathbb{R}_{+}$, $\mathcal{B}(\Gamma)$-measurable), so has $\mu$ as an invariant measure.

2) $\mathbf{M}$ from 1) is up to $\mu$-equivalence ( $c f$. [25, Definition 6.3]) unique between all Hunt processes $\mathbf{M}^{\prime}=\left(\mathbf{\Omega}^{\prime}, \mathbf{F}^{\prime},\left(\mathbf{F}_{t}^{\prime}\right)_{t \geq 0},\left(\boldsymbol{\Theta}_{t}^{\prime}\right)_{t \geq 0},\left(\mathbf{X}^{\prime}(t)\right)_{t \geq 0},\left(\mathbf{P}_{\gamma}^{\prime}\right)_{\gamma \in \Gamma}\right)$ on $\Gamma$ having $\mu$ as invariant measure and solving the martingale problem for $(-H, D(H))$, i.e., for all $G \in D(H)$

$$
\widetilde{G}\left(\mathbf{X}^{\prime}(t)\right)-\widetilde{G}\left(\mathbf{X}^{\prime}(0)\right)+\int_{0}^{t}(H G)\left(\mathbf{X}^{\prime}(s)\right) d s, \quad t \geq 0,
$$

is an $\left(\mathbf{F}_{t}^{\prime}\right)$-martingale under $\mathbf{P}_{\gamma}^{\prime}$ for $\mathcal{E}$-q.e. $\gamma \in \Gamma$. (Here, $\widetilde{G}$ denotes an $\mathcal{E}$-quasicontinuous version of $G$, cf. [25, Ch. IV, Proposition 3.3].)

Remark 3.1 In Theorem 3.1, $\mathbf{M}$ can be taken canonical, i.e., $\Omega$ is the set of all cadlag functions $\omega:[0, \infty) \rightarrow \Gamma$ (i.e., $\omega$ is right continuous on $[0, \infty)$ and has left limits on $(0, \infty)), \mathbf{X}(t)(\omega):=\omega(t), t \geq 0, \omega \in \boldsymbol{\Omega},\left(\mathbf{F}_{t}\right)_{t \geq 0}$ together with $\mathbf{F}$ is the corresponding minimum completed admissible family (cf. [10, Section 4.1]) and $\boldsymbol{\Theta}_{t}, t \geq 0$, are the corresponding natural time shifts.

Proof of Theorem 3.1. The first part of the theorem follows from Lemmas 3.3-3.5, the fact that $1 \in D(\mathcal{E}), \mathcal{E}(1,1)=0$, and [25, Chap. IV, Theorem 3.5 and Chap. V, Proposition 2.15]. The second part follows directly from (the proof of) [2, Theorem 3.5].

Let us now derive an explicit formula for the generator of $\mathcal{E}$. However, this can only be done under stronger conditions on the coefficient $c(x, y, \gamma)$.

Using (2.1) and (2.2), we have, for $F \in \mathcal{F} C_{\mathrm{b}}\left(C_{0}(X), \Gamma\right)$,

$$
\begin{aligned}
& \mathcal{E}(F)=\int_{\Gamma} \mu(d \gamma) \int_{X} z m(d y) \exp [-E(y, \gamma)+E(y, \gamma)] \int_{X} \gamma(d x) c(x, y, \gamma)\left(D_{x y}^{-+} F\right)(\gamma)^{2} \\
= & \int_{\Gamma} \mu(d \gamma) \int_{X} \gamma(d y) \exp [E(y, \gamma \backslash y)] \int_{X}(\gamma \backslash y)(d x) c(x, y, \gamma \backslash y)(F(\gamma \backslash x)-F(\gamma \backslash y))^{2} \\
= & \int_{\Gamma} \mu(d \gamma) \int_{X} \gamma(d x) \int_{X}(\gamma \backslash x)(d y) \exp [E(y, \gamma \backslash y)] c(x, y, \gamma \backslash y)(F(\gamma \backslash x)-F(\gamma \backslash y))^{2} \\
= & \int_{\Gamma} \mu(d \gamma) \int_{X} z m(d x) \exp [-E(x, \gamma)] \int_{X} \gamma(d y) \exp [E(y, \gamma \backslash y \cup x)]
\end{aligned}
$$




$$
\begin{aligned}
& \times c(x, y, \gamma \backslash y \cup x)(F(\gamma)-F(\gamma \backslash y \cup x))^{2} \\
= & \int_{\Gamma} \mu(d \gamma) \int_{X} \gamma(d x) \int_{X} z m(d y) c(y, x, \gamma \backslash x \cup y) \\
\times & \exp [-E(y, \gamma)+E(x, \gamma \backslash x \cup y)]\left(D_{x y}^{-+} F\right)(\gamma)^{2} .
\end{aligned}
$$

By (3.2) and (3.14), we have, for any $F, G \in \mathcal{F} C_{\mathrm{b}}\left(C_{0}(X), \Gamma\right)$,

$$
\mathcal{E}(F, G)=\int_{\Gamma} \mu(d \gamma) \int_{X} \gamma(d x) \int_{X} z m(d y) \tilde{c}(x, y, \gamma)\left(D_{x y}^{-+} F\right)(\gamma)\left(D_{x y}^{-+} G\right)(\gamma),
$$

where

$$
\tilde{c}(x, y, \gamma)=\frac{1}{2}(c(x, y, \gamma)+c(y, x, \gamma \backslash x \cup y) \exp [-E(y, \gamma)+E(x, \gamma \backslash x \cup y)]) .
$$

As easily seen, $\tilde{c}$ again satisfies the condition (3.1). Furthermore, $\tilde{c}$ evidently satisfies the following identity:

$$
\tilde{c}(x, y, \gamma)=\tilde{c}(y, x, \gamma \backslash x \cup y) \exp [-E(y, \gamma)+E(x, \gamma \backslash x \cup y)]
$$

so that $\tilde{\tilde{c}}=\tilde{c}$.

Theorem 3.2 Assume that, for each compact $\Lambda \subset X$,

$$
\int_{X} \gamma(d x) \int_{X} z m(d y) \tilde{c}(x, y, \gamma)\left(\mathbf{1}_{\Lambda}(x)+\mathbf{1}_{\Lambda}(y)\right) \in L^{2}(\Gamma, \mu)
$$

Then

$$
\mathcal{E}(F, K)=\int_{\Gamma}(H F)(\gamma) G(\gamma) \mu(d \gamma), \quad F, G \in \mathcal{F} C_{\mathrm{b}}\left(C_{0}(X), \Gamma\right)
$$

where

$$
(H F)(\gamma)=-2 \int_{X} \gamma(d x) \int_{X} z m(d y) \tilde{c}(x, y, \gamma)\left(D_{x y}^{-+} F\right)(\gamma) \quad \mu \text {-a.e. }
$$

and $H F \in L^{2}(\Gamma, \mu)$. The Friedrichs' extension of the operator $\left(H, \mathcal{F} C_{\mathrm{b}}\left(C_{0}(X), \Gamma\right)\right)$ in $L^{2}(\Gamma, \mu)$ is $(H, D(H))$.

Proof. Formulas (3.17) and (3.18) follow from (2.1) and (3.15), analogously to (3.14). The fact that $H F \in L^{2}(\Gamma, \mu)$ trivially follows from (3.16). 


\section{Examples}

Throughout this section, we shall always assume that a pair potential $\phi$, an activity $z$, and a corresponding Gibbs measure $\mu \in \mathcal{G}(z, E)$ are either as in Theorem 2.1 or as in Theorem 2.2. Furthermore, in the case $X=\mathbb{R}^{d}$, we shall also suppose that the condition of Lemma 2.1 is satisfied. Thus, in any case we have that $\phi$ is bounded from below, satisfies (I), and

$$
\sum_{y \in \gamma}|\phi(x, y)|<\infty \quad \text { for } m \otimes \mu \text {-a.e. }(x, \gamma) \in X \times \Gamma .
$$

By (2.1), the latter easily implies that, for $\mu$-a.e. $\gamma \in \Gamma$ and for each $x \in \gamma$,

$$
\sum_{y \in \gamma \backslash x}|\phi(x, y)|<\infty
$$

We shall now consider some examples of the coefficient $c(x, y, \gamma)$ for which the above assumptions are satisfied.

Let $a: X^{2} \rightarrow[0, \infty]$ be a symmetric measurable function such that

$$
\sup _{x \in \Lambda} \int_{X} a(x, y) m(d y)<\infty, \quad \sup _{y \in \Lambda} \int_{X} a(x, y) m(d x)<\infty
$$

for any compact $\Lambda \subset X$.

Remark 4.1 In the case $X=\mathbb{R}^{d}$, it is natural to suppose that the function $a$ is translation invariant, i.e., $a(x, y)=\tilde{a}(x-y)$ for some $\tilde{a}: X \rightarrow[0, \infty], \tilde{a}(-x)=\tilde{(} x)$, $x \in \mathbb{R}^{d}$, in which case (4.1) is equivalent to the integrability of $\tilde{a}$.

For $s \in[0,1]$ we define

$$
c(x, y, \gamma)=c_{s}(x, y, \gamma):=a(x, y) \exp [s E(x, \gamma \backslash x)-(1-s) E(y, \gamma \backslash x)]
$$

We evidently have $\tilde{c}_{s}(x, y, \gamma)=c_{s}(x, y, \gamma)$.

Proposition 4.1 1) For each $s \in[0,1]$, the coefficient $c_{s}$ satisfies (3.1).

2) Assume that the function $a$ is bounded. Then, for each $s \in[0,1 / 2]$, the coefficient $c_{s}$ satisfies (3.16). Furthermore, for each $s \in(1 / 2,1],(3.16)$ is satisfied if additionally

$$
\sup _{x \in X} \int_{X}|\exp [(2 s-1) \phi(x, y)]-1| m(d y)<\infty .
$$


Proof. 1) We have

$$
\begin{aligned}
\int_{\Gamma} \mu(d \gamma) \int_{X} \gamma(d x) \int_{X} m(d y) a(x, y) \\
\quad \times \exp [s E(x, \gamma \backslash x)-(1-s) E(y, \gamma \backslash x)]\left(\mathbf{1}_{\Lambda}(x)+\mathbf{1}_{\Lambda}(y)\right) \\
=\int_{\Gamma} \mu(d \gamma) \int_{X} z m(d x) \int_{X} m(d y) a(x, y) \\
\quad \times \exp [(s-1) E(x, \gamma)+(s-1) E(y, \gamma)])]\left(\mathbf{1}_{\Lambda}(x)+\mathbf{1}_{\Lambda}(y)\right) \\
=\int_{X} z m(d x) \int_{X} m(d y) a(x, y)\left(\mathbf{1}_{\Lambda}(x)+\mathbf{1}_{\Lambda}(y)\right) \\
\quad \times \int_{\Gamma} \mu(d \gamma) \prod_{u \in \gamma}(1+(\exp [(s-1) \phi(x, u)+(s-1) \phi(y, u)]-1)) \\
=\int_{X} z m(d x) \int_{X} m(d y) a(x, y)\left(\mathbf{1}_{\Lambda}(x)+\mathbf{1}_{\Lambda}(y)\right) \\
\quad \times \int_{\Gamma} \mu(d \gamma)\left(1+\sum_{n=1}^{\infty} \sum_{\left\{u_{1}, \ldots, u_{n}\right\} \subset \gamma} \prod_{i=1}^{n}\left(\exp \left[(s-1) \phi\left(x, u_{i}\right)+(s-1) \phi\left(y, u_{i}\right)\right]-1\right)\right) \\
=\int_{X} z m(d x) \int_{X} m(d y) a(x, y)\left(\mathbf{1}_{\Lambda}(x)+\mathbf{1}_{\Lambda}(y)\right) \\
\quad \times \int_{\Gamma} \mu(d \gamma)\left(1+\sum_{n=1}^{\infty} \frac{1}{n !} \int_{X^{n}} \prod_{i=1}^{n}\left(\exp \left[(s-1) \phi\left(x, u_{i}\right)+(s-1) \phi\left(y, u_{i}\right)\right]-1\right)\right. \\
\left.\quad \times k_{\mu}^{(n)}\left(u_{1}, \ldots, u_{n}\right) m\left(d u_{1}\right) \ldots m\left(d u_{n}\right)\right) .
\end{aligned}
$$

Using the Ruelle bound, we get, for any $x, y \in X$,

$$
\begin{aligned}
\int_{X^{n}} & \prod_{i=1}^{n}\left|\exp \left[(s-1) \phi\left(x, u_{i}\right)+(s-1) \phi\left(y, u_{i}\right)\right]-1\right| \\
& \times k_{\mu}^{(n)}\left(u_{1}, \ldots, u_{n}\right) m\left(d u_{1}\right) \cdots m\left(d u_{n}\right) \\
\leq & \left(\xi \int_{X}|\exp [(s-1) \phi(x, u)+(s-1) \phi(y, u)]-1| m(d u)\right)^{n} \\
\leq & \left(\xi \int_{X}|\exp [-\phi(x, u)-\phi(y, u)]-1| m(d u)\right)^{n} \\
\leq & \left(\xi \int_{X}(|\exp [-\phi(x, u)]-1|+\exp [-\phi(x, u)]|\exp [-\phi(y, u)]-1|) m(d u)\right)^{n} .
\end{aligned}
$$

From here, (I) and (4.4), the statement follows. 
2) Analogously, we have:

$$
\begin{aligned}
& \int_{\Gamma} \mu(d \gamma)\left(\int_{X} \gamma(d x) \int_{X} z m(d y) c_{s}(x, y, \gamma)\left(\mathbf{1}_{\Lambda}(x)+\mathbf{1}_{\Lambda}(y)\right)\right)^{2} \\
& =\int_{\Gamma} \mu(d \gamma) \int_{X} \gamma(d x) \int_{X} z m(d y) \int_{X} z m\left(d y^{\prime}\right) c_{s}(x, y, \gamma) c_{s}\left(x, y^{\prime}, \gamma\right) \\
& \times\left(\mathbf{1}_{\Lambda}(x)+\mathbf{1}_{\Lambda}(y)\right)\left(\mathbf{1}_{\Lambda}(x)+\mathbf{1}_{\Lambda}\left(y^{\prime}\right)\right) \\
& +\int_{\Gamma} \mu(d \gamma) \int_{X} \gamma(d x) \int_{X}(\gamma \backslash x)\left(d x^{\prime}\right) \int_{X} z m(d y) \int_{X} z m\left(d y^{\prime}\right) \\
& \times c_{s}(x, y, \gamma) c_{s}\left(x^{\prime}, y^{\prime}, \gamma\right)\left(\mathbf{1}_{\Lambda}(x)+\mathbf{1}_{\Lambda}(y)\right)\left(\mathbf{1}_{\Lambda}\left(x^{\prime}\right)+\mathbf{1}_{\Lambda}\left(y^{\prime}\right)\right) \\
& =\int_{\Gamma} \mu(d \gamma) \int_{X} z m(d x) \int_{X} z m(d y) \int_{X} z m\left(d y^{\prime}\right) \exp [-E(x, \gamma)] \\
& \times c_{s}(x, y, \gamma \cup x) c_{s}\left(x, y^{\prime}, \gamma \cup x\right)\left(\mathbf{1}_{\Lambda}(x)+\mathbf{1}_{\Lambda}(y)\right)\left(\mathbf{1}_{\Lambda}(x)+\mathbf{1}_{\Lambda}\left(y^{\prime}\right)\right) \\
& +\int_{\Gamma} \mu(d \gamma) \int_{X} z m(d x) \int_{X} z m\left(d x^{\prime}\right) \int_{X} z m(d y) \int_{X} z m\left(d y^{\prime}\right) \\
& \times \exp \left[-E(x, \gamma)-E\left(x^{\prime}, \gamma\right)-\phi\left(x, x^{\prime}\right)\right] \\
& \times c_{s}\left(x, y, \gamma \cup x \cup x^{\prime}\right) c_{s}\left(x^{\prime}, y^{\prime}, \gamma \cup x \cup x^{\prime}\right)\left(\mathbf{1}_{\Lambda}(x)+\mathbf{1}_{\Lambda}(y)\right)\left(\mathbf{1}_{\Lambda}\left(x^{\prime}\right)+\mathbf{1}_{\Lambda}\left(y^{\prime}\right)\right) \\
& =\int_{X} z m(d x) \int_{X} z m(d y) \int_{X} z m\left(d y^{\prime}\right) a(x, y) a\left(x, y^{\prime}\right)\left(\mathbf{1}_{\Lambda}(x)+\mathbf{1}_{\Lambda}(y)\right)\left(\mathbf{1}_{\Lambda}(x)+\mathbf{1}_{\Lambda}\left(y^{\prime}\right)\right) \\
& \times \int_{\Gamma} \mu(d \gamma) \exp \left[\sum_{u \in \gamma}\left((2 s-1) \phi(x, u)-(1-s) \phi(y, u)-(1-s) \phi\left(y^{\prime}, u\right)\right)\right] \\
& +\int_{X} z m(d x) \int_{X} z m\left(d x^{\prime}\right) \int_{X} z m(d y) \int_{X} z m\left(d y^{\prime}\right) a(x, y) a\left(x^{\prime}, y^{\prime}\right) \\
& \times\left(\mathbf{1}_{\Lambda}(x)+\mathbf{1}_{\Lambda}(y)\right)\left(\mathbf{1}_{\Lambda}\left(x^{\prime}\right)+\mathbf{1}_{\Lambda}\left(y^{\prime}\right)\right) \\
& \times \exp \left[(2 s-1) \phi\left(x, x^{\prime}\right)-(1-s) \phi\left(x^{\prime}, y\right)-(1-s) \phi\left(x, y^{\prime}\right)\right] \\
& \times \int_{\Gamma} \mu(d \gamma) \exp \left[\sum_{u \in \gamma}-(1-s)\left(\phi(x, u)+\phi\left(x^{\prime}, u\right)+\phi(y, u)+\phi\left(y^{\prime}, u\right)\right)\right] \\
& \leq C_{2}\left(\int_{X} z m(d x) \int_{X} z m(d y) \int_{X} z m\left(d y^{\prime}\right) a(x, y) a\left(x, y^{\prime}\right)\left(\mathbf{1}_{\Lambda}(x)+\mathbf{1}_{\Lambda}(y)\right)\left(\mathbf{1}_{\Lambda}(x)+\mathbf{1}_{\Lambda}\left(y^{\prime}\right)\right)\right. \\
& \times \exp \left[\xi \sup _{x \in X} \sup _{y \in X} \sup _{y^{\prime} \in X} \int_{X} \mid \exp [(2 s-1) \phi(x, u)\right. \\
& \left.\left.-(1-s) \phi(y, u)-(1-s) \phi\left(y^{\prime}, u\right)\right]-1 \mid m(d u)\right] \\
& +\int_{X} z m(d x) \int_{X} z m\left(d x^{\prime}\right) \int_{X} z m(d y) \int_{X} z m\left(d y^{\prime}\right) a(x, y) a\left(x^{\prime}, y^{\prime}\right) \\
& \times\left(\mathbf{1}_{\Lambda}(x)+\mathbf{1}_{\Lambda}(y)\right)\left(\mathbf{1}_{\Lambda}\left(x^{\prime}\right)+\mathbf{1}_{\Lambda}\left(y^{\prime}\right)\right) \exp \left[(2 s-1) \phi\left(x, x^{\prime}\right)\right]
\end{aligned}
$$




$$
\begin{gathered}
\times \exp \left[\xi \sup _{x \in X} \sup _{x^{\prime} \in X} \sup _{y \in X} \sup _{y^{\prime} \in X} \int_{X} \mid \exp \left[-(1-s)\left(\phi(x, u)+\phi\left(x^{\prime}, u\right)\right.\right.\right. \\
\left.\left.\left.\left.\quad+\phi(y, u)+\phi\left(y^{\prime}, u\right)\right)\right]-1 \mid m(d u)\right]\right) \\
\leq C_{3}\left(\int_{X} z m(d x) \int_{X} z m(d y) \int_{X} z m\left(d y^{\prime}\right) a(x, y) a\left(x, y^{\prime}\right)\left(\mathbf{1}_{\Lambda}(x)+\mathbf{1}_{\Lambda}(y)\right)\left(\mathbf{1}_{\Lambda}(x)+\mathbf{1}_{\Lambda}\left(y^{\prime}\right)\right)\right. \\
+\int_{X} z m(d x) \int_{X} z m\left(d x^{\prime}\right) \int_{X} z m(d y) \int_{X} z m\left(d y^{\prime}\right) a(x, y) a\left(x^{\prime}, y^{\prime}\right) \\
\times\left(\mathbf{1}_{\Lambda}(x)+\mathbf{1}_{\Lambda}(y)\right)\left(\mathbf{1}_{\Lambda}\left(x^{\prime}\right)+\mathbf{1}_{\Lambda}\left(y^{\prime}\right)\right)\left|\exp \left[(2 s-1) \phi\left(x, x^{\prime}\right)\right]-1\right| \\
\left.+\left(\int_{X} z m(d x) \int_{X} z m(d y) a(x, y)\left(\mathbf{1}_{\Lambda}(x)+\mathbf{1}_{\Lambda}(y)\right)\right)^{2}\right),
\end{gathered}
$$

where $C_{2}, C_{3}>0$. Using (I), (4.1), (4.3), and the boundedness of $a$, we easily conclude that the expression in (4.5) is finite. Indeed, for example, we have:

$$
\begin{aligned}
\int_{X} z m(d x) \int_{X} z m\left(d x^{\prime}\right) \int_{\Lambda} z m(d y) \int_{\Lambda} z m\left(d y^{\prime}\right) a(x, y) a\left(x^{\prime}, y^{\prime}\right)\left|\exp \left[(2 s-1) \phi\left(x, x^{\prime}\right)\right]-1\right| \\
\leq\left(\sup _{(u, v) \in X^{2}} a(u, v)\right) \int_{\Lambda} z m(d y) \int_{\Lambda} z m\left(d y^{\prime}\right) \\
\quad \times \int_{X} z m(d x) a(x, y) \int_{X} z m\left(d x^{\prime}\right)\left|\exp \left[(2 s-1) \phi\left(x, x^{\prime}\right)\right]-1\right|<\infty .
\end{aligned}
$$

Thus, the proposition is proved.

Let us now present a straightforward generalization of the above result. Let now $a: X^{2} \rightarrow \mathbb{R}$ be a measurable function which satisfies (4.1) (and which is not necessarily symmetric). For $u, v \in[0,1]$, we define

$$
\varkappa(x, y, \gamma)=\varkappa_{u, v}(x, y, \gamma):=\exp [u E(x, \gamma \backslash x)-(1-v) E(y, \gamma)]
$$

and

$$
c(x, y, \gamma)=c_{u, v}(x, y, \gamma):=a(x, y) \varkappa_{u, v}(x, y, \gamma) .
$$

In particular, for $u=v$, we get the previous example of a Kawasaki dynamics. Note also that, for $u=0$ and $v=1$, we get

$$
c_{0,1}(x, y, \gamma)=a(x, y)
$$

By (3.15), we have

$$
\begin{aligned}
\tilde{c}_{u, v}(x, y, \gamma)=\frac{1}{2} & (a(x, y) \exp [u E(x, \gamma \backslash x)-(1-v) E(y, \gamma)] \\
& +a(y, x) \exp [v E(x, \gamma \backslash x)-(1-u) E(y, \gamma)]) .
\end{aligned}
$$

Absolutely analogously to Proposition 4.1, one can prove its following generalization. 
Proposition 4.2 1) For each $u, v \in[0,1]$, the coefficient $c_{u, v}$ satisfies (3.1).

2) Assume that the function a is bounded. Then, (3.16) is satisfied if

$$
\sup _{x \in X} \int_{X}|\exp [(2(u \vee v)-1) \phi(x, y)]-1| m(d y)<\infty .
$$

\section{Scaling limits of Kawasaki dynamics}

We start this section with a brief discussion of Glauber dynamics of continuous particle systems.

\subsection{Glauber (birth-and-death) dynamics}

In the classical Ising model, the Glauber dynamics means that particles randomly change their spin value, which is called a spin-flip. The generator of this dynamics is given by

$$
\left(H_{\mathrm{G}} f\right)(\sigma)=\sum_{x \in \mathbb{Z}^{d}} a(x, \sigma)\left(\nabla_{x} f\right)(\sigma),
$$

where

$$
\left(\nabla_{x} f\right)(\sigma)=f\left(\sigma^{x}\right)-f(\sigma)
$$

$\sigma^{x}$ denoting the configuration $\sigma$ in which the particle at site $x$ has changed its spin value. In the interpretation of a lattice system with spin space $S=\{-1,1\}$ as a model of a lattice gas, the Glauber dynamics means that, at each site $x$, a particle randomly appears and disappears. Hence, this dynamics may be interpreted as a birth-and-death process on $\mathbb{Z}^{d}$. Therefore, in the continuous case, an analog of the Glauber dynamics should be a process in which particles randomly appear and disappear in the space, i.e., a spatial birth-and-death process. The generator of such a process is informally given by the formula

$$
\left(H_{\mathrm{G}} F\right)(\gamma)=\sum_{x \in \gamma} d(x, \gamma)\left(D_{x}^{-} F\right)(\gamma)+\int_{\mathbb{R}^{d}} b(x, \gamma)\left(D_{x}^{+} F\right)(\gamma) d x,
$$

where

$$
\left(D_{x}^{-} F\right)(\gamma)=F(\gamma \backslash x)-F(\gamma), \quad\left(D_{x}^{+} F\right)(\gamma)=F(\gamma \cup x)-F(\gamma) .
$$

Spatial birth-and-death processes were first discussed by Preston in [28]. Under some conditions on the birth and death rates, Preston proved the existence of such processes in a bounded domain in $\mathbb{R}^{d}$. Though the number of particles can be arbitrarily large in this case, the total number of particles remains finite at any moment of time.

The problem of construction of a spatial birth-and-death process in the infinite volume was initiated by Holley and Stroock in [15]. In fact, in that paper, birthand-death processes in bounded domains were analyzed in detail. Only in a very 
special case of nearest neighbor birth-and-death processes on the real line, the existence of a corresponding process on the whole space was proved and its properties were studied. In [11], Glötzl derived conditions on the coefficients $d(x, \gamma), b(x, \gamma)$ under which the Glauber generator becomes a symmetric operator in the space $L^{2}(\mu)$, where $\mu$ is a given Gibbs measure. Let us also mention the papers [3, 33] devoted to the study of the spectral gap of the Glauber dynamics in the finite volume, for which the death coefficient is equal to 1 . An analog of such a dynamics, but on the whole space (thus, involving infinite configurations), was constructed in [18]. The coefficients of the generator of this dynamics are given by

$$
d(x, \gamma)=1, \quad b(x, \gamma)=\exp [-E(y, \gamma)]
$$

and this dynamics has a Gibbs measure corresponding to the pair potential $\phi$ as symmetrizing measure. The result about the spectral gap for a positive $\phi$ has also been extended in [18] to the infinite volume. We also refer to [13] for a discussion of a scaling limit of equilibrium fluctuations of this dynamics.

By analogy with the Kawasaki dynamics, we are now able to construct an equilibrium Kawasaki dynamics in the general case. So, we consider a measurable mapping

$$
X \times \Gamma \ni(x, \gamma) \mapsto d(x, \gamma) \in[0, \infty)
$$

and assume that, for each compact $\Lambda \subset X$,

$$
\int_{\Gamma} \mu(d \gamma) \int_{\Lambda} \gamma(d x) d(x, \gamma)<\infty
$$

We define a bilinear form

$$
\mathcal{E}_{\mathrm{G}}(F, G):=\int_{\Gamma} \mu(d \gamma) \int_{X} \gamma(d x) d(x, \gamma)\left(D_{x}^{-} F\right)(\gamma)\left(D_{x}^{-} G\right)(\gamma)
$$

where $F, G \in \mathcal{F} C_{\mathrm{b}}\left(C_{0}(X), \Gamma\right)$. This bilinear form is closable on $L^{2}(\Gamma, \mu)$, and its closure will be denoted by $\left(\mathcal{E}_{\mathrm{G}}, D\left(\mathcal{E}_{\mathrm{G}}\right)\right)$. The latter is a quasi-regular Dirichlet form on $L^{2}(\ddot{\Gamma}, \mu)$. Furthermore, the set $\ddot{\Gamma} \backslash \Gamma$ is $\mathcal{E}_{\mathrm{G}}$-exceptional. Therefore, there exists a conservative Hunt process which is properly associated with $\left(\mathcal{E}_{\mathrm{G}}, D\left(\mathcal{E}_{\mathrm{G}}\right)\right)$.

Next, assume that, for each compact $\Lambda \subset X$,

$$
\begin{gathered}
\int_{\Lambda} \gamma(d x) d(x, \gamma) \in L^{2}(\Gamma, \mu) \\
\int_{\Lambda} z m(d x) b(x, \gamma) \in L^{2}(\Gamma, \mu),
\end{gathered}
$$

where

$$
b(x, \gamma):=\exp [-E(x, \gamma)] d(x, \gamma \cup x), \quad x \in X, \gamma \in \Gamma .
$$


Then, $\mathcal{F} C_{\mathrm{b}}\left(C_{0}(X), \Gamma\right)$ is a subset of the domain of the generator $H_{\mathrm{G}}$ of the Dirichlet form $\left(\mathcal{E}_{\mathrm{G}}, D\left(\mathcal{E}_{\mathrm{G}}\right)\right)$, and for each $F \in \mathcal{F} C_{\mathrm{b}}\left(C_{0}(X), \Gamma\right)$,

$$
\left(H_{\mathrm{G}} F\right)(\gamma)=-\int_{X} z m(d x) b(x, \gamma)\left(D_{x}^{+} F\right)(\gamma)-\int_{X} \gamma(d x) d(x, \gamma)\left(D_{x}^{-} F\right)(\gamma) \quad \mu \text {-a.e. }
$$

Also by analogy with the Kawasaki dynamics, one can construct the following examples of the Glauber dynamics (the Gibbs measure $\mu$ being the same as in Section 4). For each $s \in[0,1]$, we define

$$
d(x, \gamma)=d_{s}(x, \gamma):=\exp [s E(x, \gamma \backslash x)]
$$

so that

$$
b(x, \gamma)=b_{s}(x, \gamma)=\exp [(s-1) E(x, \gamma)] .
$$

Then, for each $s \in[0,1]$, (5.1) holds, and therefore the corresponding Glauber dynamics exists. Furthermore, for each $s \in[0,1 / 2]$, the coefficients $d_{s}, b_{s}$ satisfy (5.2), while for $s \in(1 / 2,1],(5.2)$ is satisfied if (4.3) holds.

Note that, though the construction of the Glauber dynamics and that of the Kawasaki dynamics look quite similar, there is a drastic difference between them in that (at least heuristically) the law of conservation of the number of particles holds for the Kawasaki dynamics, and does not for the Glauber dynamics. We, therefore, cannot expect a spectral gap for the generator of the Kawasaki dynamics in the infinite volume.

Furthermore, the Glauber and Kawasaki dynamics have different sets of symmetrizing measures. Indeed, the set of symmetrizing measures of a given Glauber dynamics consists of all grand-canonical Gibbs measures corresponding to a given relative energy of interaction and a fixed activity parameter $z>0$, while for a given Kawasaki dynamics activity parameter $z>0$ may be arbitrary.

\subsection{Glauber dynamics as a limiting Kawasaki dynamics}

Let $X=\mathbb{R}^{d}$ and let $\mu$ be a Gibbs measure as in Theorem 2.2, 1) (low activity-high temperature regime). We fix a function $\tilde{a}: \mathbb{R}^{d} \rightarrow[0, \infty)$ such that $\tilde{a}(-x)=\tilde{a}(x)$, $x \in \mathbb{R}^{d}$, and $\tilde{a} \in L^{1}\left(\mathbb{R}^{d}, d x\right)$. For each $s \in[0,1]$, consider the Kawasaki dynamics corresponding to the coefficient $c=c_{s}$ given by $(4.2)$ with $a(x, y):=\tilde{a}(x-y)$.

Let us now consider the following scaling of this dynamics. For each $\delta>0$, define

$$
\tilde{a}_{\delta}(x)=\delta^{d} \tilde{a}(\delta \cdot), \quad x \in \mathbb{R}^{d},
$$

and consider the $(\delta, s)$-Kawasaki dynamics which is defined just as the dynamics above, but by using the function $\tilde{a}_{\delta}$ instead of $\tilde{a}$. Denote by $\left(H_{\delta, s}, D\left(H_{\delta, s}\right)\right)$ the generator of this dynamics. 
Let us also fix the $s$-Glauber dynamics corresponding to

$$
\begin{aligned}
& d(x, \gamma)=d_{s}(x, \gamma):=\alpha \exp [s E(x, \gamma \backslash x)] \\
& b(x, \gamma)=b_{s}(x, \gamma)=\alpha \exp [(s-1) E(x, \gamma)],
\end{aligned}
$$

where $s \in[0,1]$ and

$$
\alpha:=2 k_{\mu}^{(1)} \int_{\mathbb{R}^{d}} a(x) d x
$$

(note that the first correlation function $k_{\mu}^{(1)}$ is a constant). Denote by $\left(H_{0, s}, D\left(H_{0, s}\right)\right.$ ) the generator of this dynamics.

We expect that the $s$-Glauber dynamics is the limit of the $(\delta, s)$-Kawasaki dynamics as $\delta \rightarrow 0$. In particular, in the case $s=0$, it is shown in [8] that, for each $\varphi \in C_{0}\left(\mathbb{R}^{d}\right)$,

$$
H_{\delta, s} e^{\langle\varphi, \cdot\rangle} \rightarrow H_{0, s} e^{\langle\varphi, \cdot\rangle} \quad \text { in } L^{2}(\Gamma, \mu)
$$

as $\delta \rightarrow 0$. In the case where the potential $\phi$ is non-negative, one can conclude from [18] that the set of finite linear combinations of the exponential functions is a core for the Glauber generator $\left(H_{0, s}, D\left(H_{0, s}\right)\right)$. From here, using a classical result from the theory of semigroups [6], one derives the weak convergence of finite-dimensional distributions of the corresponding equilibrium dynamics, starting with their equilibrium distribution $\mu$.

\subsection{Diffusion approximation for the Kawasaki dynamics}

Now, let $X=\mathbb{R}^{d}$ and let $\mu$ be a Gibbs measure as in Theorem 2.2. We will consider a Kawasaki dynamics as in subsec. 5.2 , but this time we will additionally assume that $\tilde{a}(x)$ only depends on $|x|$, and has compact support. We again consider the corresponding $(\delta, s)$-Kawasaki dynamics, but this time we are interested in its limiting behavior as $\delta \rightarrow \infty$. It appears that, we additionally have to re-scale time by multiplying it by $\delta^{2}$. Thus, the generator of this dynamics is given by $\widetilde{H}_{\delta, s}=\delta^{2} H_{\delta, s}$. Under quite weak assumptions on the potential $\phi$, it is shown in [17] that, for each function $F$ from some set of smooth local functions on the configuration space,

$$
\widetilde{H}_{\delta, s} F \rightarrow \widetilde{H}_{s} F \quad \text { in } L^{2}(\Gamma, \mu)
$$

as $\delta \rightarrow \infty$. Here $\widetilde{H}_{s}$ is the generator of a diffusion dynamics given by

$$
\begin{aligned}
\widetilde{H}_{s} F(\gamma)= & c \int_{\mathbb{R}^{d}} \gamma(d x)\left(-\Delta_{x} F(\gamma)+2 \sum_{u \in \gamma \backslash x}\left\langle\nabla_{x} F(\gamma), s \nabla \phi(x-u)\right\rangle\right) \\
& \times \exp [(-2 s+1) E(x, \gamma \backslash x)],
\end{aligned}
$$


where

$$
c:=z \int_{\mathbb{R}^{d}} a(x)\left(x^{1}\right)^{2} d x
$$

$\left(x^{1}\right.$ denoting the first coordinate of $\left.x \in \mathbb{R}^{d}\right), \Delta_{x} F(\gamma):=\left.\Delta_{y} F(\gamma \backslash x \cup y)\right|_{y=x}$, and $\nabla_{x} F(\gamma)$ is defined by analogy. In particular, for $s=1 / 2$,

$$
\widetilde{H}_{s} F(\gamma)=c \int_{\mathbb{R}^{d}} \gamma(d x)\left(-\Delta_{x} F(\gamma)+\sum_{u \in \gamma \backslash x}\left\langle\nabla_{x} F(\gamma), \nabla \phi(x-u)\right\rangle\right),
$$

which is the generator of the gradient stochastic dynamics, e.g. [1, 9].

In the case where $\mu$ is a Gibbs measure as in Theorem $2.2,3), \tilde{\phi} \in C_{\mathrm{b}}^{3}\left(\mathbb{R}^{d}\right)$, and $\tilde{\phi}$ sufficiently quickly converges to zero at infinity, Choi, Park, and Yoo [4] found a core for the generator of the gradient stochastic dynamics. Using this result, we derive the weak convergence of finite-dimensional distributions of the corresponding equilibrium dynamics, starting with their equilibrium distribution $\mu$.

\section{Acknowledgements}

The authors acknowledge the financial support of the DFG Forschergruppe "Spectral analysis, asymptotic distributions and stochastic dynamics" and SFB 701 "Spectral structures and topological methods in mathematics". Yu.K. was partially supported by the DFG research grant TR120/12-1. E.L. acknowledges the financial support of the SFB 611, Bonn University.

\section{References}

[1] S. Albeverio, Yu. G. Kondratiev, and M. Röckner, Analysis and geometry on configuration spaces. The Gibbsian case, J. Func. Anal. 157 (1998) 242-291.

[2] S. Albeverio and M. Röckner, Dirichlet form methods for uniqueness of martigale problems and applications, in "Stochastic Analysis. Proceedings of Symposia in Pure Mathematics, Vol. 57", M. C. Cranston and M. A. Pinsky ed., Am. Math. Soc., 1995, pp. 513-528.

[3] L. Bertini, N. Cancrini, and F. Cesi, The spectral gap for a Glauber-type dynamics in a continuous gas, Ann. Inst. H. Poincaré Probab. Statist. 38 (2002) 91-108.

[4] V. Choi, Y. M. Park, and H. J. Yoo, Dirichlet forms and Dirichlet operators for infinite particle systems: essential self-adjointness, J. Math. Phys. 39 (1998) 6509-6536.

[5] D. J. Daley and D. Vere-Jones, "An Introduction to the Theory of Point Processes", Springer-Verlag, 1988. 
[6] E. B. Davies, "One-Parameter Semigroups", Academic Press, London, 1980.

[7] A. De Masi and E. Presutti, "Mathematical Methods for Hydrodynamic Limits", Lecture Notes in Mathematics, Vol. 1501, Springer-Verlag, 1991.

[8] D. L. Finkelshtein,Yu. G. Kondratiev, and E. W. Lytvynov, Glauber dynamics as a scaling limit of Kawasaki dynamics, Preprint, 2006.

[9] J. Fritz, Gradient dynamics of infinite point systems, Ann. Prob. 15 (1987) 478514.

[10] M. Fukushima, "Dirichlet Forms and Symmetric Markov Processes", NorthHolland, 1980.

[11] E. Glötzl, Time reversible and Gibbsian point processes. I. Markovian spatial birth and death processes on a general phase space, Math. Nachr. 102 (1981) 217-222.

[12] E. Glötzl, Time reversible and Gibbsian point processes. II. Markovian particle jump processes on a general phase space, Math. Nachr. 106 (1982) 63-71.

[13] M. Grothaus, Scaling limit of interacting spatial birth and death processes in continuous systems, J. Funct. Anal. 239 (2006) 414-445.

[14] M. Grothaus, Yu. G. Kondratiev, E. Lytvynov, and M. Röckner, Scaling limit of stochastic dynamics in classical continuous systems, Ann. Prob. 31 (2003), 14941532 .

[15] R. A. Holley and D. W. Stroock, Nearest neighbor birth and death processes on the real line, Acta Math. 140 (1978) 103-154.

[16] Yu. G. Kondratiev, T. Kuna, and J. L. Silva, Marked Gibbs measures via cluster expansion, Methods Funct. Anal. Topology 4 (1998), no. 4, 50-81.

[17] Yu. G. Kondratiev, O. V. Kutoviy, and E. W. Lytvynov, Diffusion approximation for Kawasaki dynamics in continuum, in preparation.

[18] Yu. G. Kondratiev and E. Lytvynov, Glauber dynamics of continuous particle systems, Ann. Inst. H. Poincaré Probab. Statist. 41 (2005) 685-702.

[19] Yu. G. Kondratiev, E. Lytvynov, and M. Röckner, Infinite interaction diffusion particles I: Equilibrium process and its scaling limit, Forum Math. 18 (2006) 9-43

[20] Yu. G. Kondratiev, E. Lytvynov, and M. Röckner, Equilibrium fluctuations for the Kawasaki dynamics of continuous particle systems, in preparation.

[21] O. Kallenberg, "Random Measures", Academic Press, 1975. 
[22] T. Kuna, "Studies in Configuration Space Analysis and Applications", Ph.D. thesis, Bonn University, 1999.

[23] T. Kuna, Properties of marked Gibbs measures in high temperature regime, Methods Funct. Anal. Topology 7 (2001), no. 3, 33-53.

[24] T. M. Liggett, "Interacting Particle Systems", Springer-Verlag, 1985.

[25] Z.-M. Ma and M. Röckner, "An Introduction to the Theory of (Non-Symmetric) Dirichlet Forms", Springer-Verlag, 1992.

[26] Z.-M. Ma and M. Röckner, Construction of diffusions on configuration spaces, Osaka J. Math. 37 (2000) 273-314.

[27] X. X. Nguyen, H. Zessin, Integral and differentiable characterizations of the Gibbs process, Math. Nachr. 88 (1979) 105-115.

[28] C. Preston, Spatial birth-and-death processes, in "Proceedings of the 40th Session of the International Statistical Institute (Warsaw, 1975), Vol. 2", Bull. Inst. Internat. Statist., Vol. 46, 1975, pp. 371-391.

[29] M. Röckner and B. Schmuland, A support property for infinite-dimensional interacting diffusion processes, C. R. Acad. Sci. Paris 326 (1998), Série I, 359-364.

[30] D. Ruelle, "Statistical Mechanics. Rigorous Results", Benjamins, 1969.

[31] D. Ruelle, Superstable interaction in classical statistical mechanics, Comm. Math. Phys. 18 (1970), 127-159.

[32] H. Spohn, "Large Scale Dynamics of Interacting Particle Systems", SpringerVerlag, 1991.

[33] L. Wu, Estimate of spectral gap for continuous gas, Ann. Inst. H. Poincaré Probab. Statist. 40 (2004), 387-409. 